\title{
AOIR
}

Selected Papers of \#AolR2021:

The 22nd Annual Conference of the

Association of Internet Researchers

Virtual Event / 13-16 Oct 2021

\section{FROM MONOPOLIZING MEMORY TO CO-CREATING IT: OPENNESS AND EQUITY IN THE DIGITAL ECOSYSTEM}

\author{
Angeliki Tzouganatou \\ University of Hamburg \\ Jennifer Krueckeberg \\ University of Hamburg
}

Today, memory, whether collective or personal, is increasingly dependent on digital media that have permeated various aspects of people's everyday lives. Particularly, the ongoing Covid-19 pandemic has highlighted people's and memory institutions' dependency on social media for their memory practices and efforts to reach the public in these challenging times. However, social media and its infrastructures do not necessarily align with the needs and interests of individuals and the wider public, or with those of memory institutions. Commercial interests embedded in social media infrastructures shape what should be remembered based on short-term business goals, that are not concerned with the preservation of data because of their historical significance, but rather, their potential profit. Drawing from ethnographic research on young people's memory practices and open knowledge practices in the digital economy, this paper investigates equitable ways to the current 'big tech' relying system. In addition, it discusses the role of community and human-centered practices as a basis to untap the potential of open public memory infrastructures that run independently from technology monopolies.

\section{Methodology}

In the doctoral research presented here, the applied methods included ethnographic fieldwork using observations and expert interviews conducted by Tzouganatou at Open Knowledge Finland to investigate economic and governance issues concerning platforms. Krueckeberg has conducted 12 months of ethnographic fieldwork into digital memory practices of young people aged between 13 - 27, living in London and several German cities. During fieldwork semi-structured interviews, photo elicitation and a digital storytelling workshop were carried out. 


\section{Co-dependencies of memory ecosystems}

Europe is mostly operating under the GAFAM (Google, Amazon, Facebook, Apple, Microsoft) ecosystem (van Dijck 2020a), where commercial platforms extract data from users through surveillance mechanisms, and monetizes data by using it as a basis for making behavioural predictions (Zuboff 2019). This is linked to the platformization of the web, where platform's thriving business model based on data surveillance has turned commercial platforms to dominant infrastructures of the internet ecosystem, and penetrated society at large (Helmond 2015; Plantin et al. 2016; van Dijck 2020b). In addition to this observation, digital personal, institutional and commercial memory archives are overlapping now (Garde-Hansen 2011) with social media playing a vital role, as these platforms guide people's behaviour through their norms (Ross 2019) and the imaginary of digital infrastructures (Markham 2020). Therefore, current digital ecosystems have an immense influence on what will be remembered.

Establishing open public digital infrastructures and digital sovereignty (Floridi 2020), is a way to envision positive futures beyond the market and business domain with a greater degree of independence. Work on co-creation (Fuster Morell and Senabre Hidalgo 2020), participatory design and co-design (Marttila and Botero 2017; Poderi 2019) directs to reposition these practices in light of platform capitalism (Srnicek 2016), and highlights the importance of fair and community governance.

\section{Envisioning independent digital futures}

The young participants of this paper have grown up with digital media and many of their early memories were made and chronicled online. However, the commercialisation of data and the proliferation of advertisements that are 'creepily' tailored towards individual preferences are leading to a growing suspicion of what happens to their data and personal memory. In addition, issues of data exploitation are increasingly visible within public discourse and pop culture, making youth more aware of the complexities (Taffel 2019) behind the technologies they use daily. Yet, young people's growing understanding of the exploitation and misuse of personal data has not resulted in large scale rebellions. Instead, due to a lack of mainstream alternatives and the convenience of the status quo, young people rely on adjusting their own behaviour of sharing information online to protect their data and memories. Nonetheless, controlling and optimizing the self is not dissolving issues of data ownership and data exploitation, but risks feeding into the favouring of memories based on their marketability instead of cultural or social importance.

Moreover, it is not only individuals whose memory practices are affected by the current ecosystem. Particularly due to the ongoing pandemic, memory institutions are striving to retain audience engagement through social media while their premises are closed. Although enabling public engagement, it is important to interrogate whether these platforms are aligned with memory institutions' values and mission. The current digital ecosystem is characterized by immense inequalities that are perpetuated by obfuscated practices like untransparent business models and proprietary algorithms for marketing and monetization purposes. Open knowledge advocates suggest that decentralized and 
distributed practices could strengthen participatory elements in which people are directly involved in the production mode and decision making process of for example related policies for the organisation and management of data. This practice of co-creating with communities could potentially better match people's needs. In addition, these practices aim to enable people's participation and foster collective memory, which could promote data ownership by individuals and communities to govern and use their data for their own benefits.

This paper proposes a fair data ecosystem to memory work, enabling a participatory approach to data governance, that builds upon the work of initiatives such as MyData and the DECODE project. This contribution aims to be an exploration for a theoretical prototype combining aspects of open public memory infrastructures, and digital sovereignty, with human-centered practices derived from ethnographic fieldwork with youth. These youth practices illuminate potentialities in how frustrations with the status quo could be used towards independence from the current business oriented ecosystem.

\section{Acknowledgments}

This work is part of the POEM (Participatory Memory Practices) project and has received funding from the European Union's Horizon 2020 research and innovation program under the Marie Skłodowska-Curie grant agreement No. 764859.

\section{References}

Floridi, Luciano (2020): The Fight for Digital Sovereignty: What It Is, and Why It Matters, Especially for the EU. In Philos. Technol. 33 (3), pp. 369-378.

Fuster Morell, Mayo; Senabre Hidalgo, Enric (2020): Co-creation applied to public policy: a case study on collaborative policies for the platform economy in the city of Barcelona. In CoDesign, pp. 1-20.

Garde-Hansen, Joanne (2011): Media and memory. Edinburgh: Edinburgh University Press.

Helmond, Anne (2015): The Platformization of the Web: Making Web Data Platform Ready. In Social Media + Society 1 (2).

Markham, Annette (2020): The limits of the imaginary: Challenges to intervening in future speculations of memory, data, and algorithms. In New Media \& Society, pp. 1-24.

Marttila, Sanna; Botero, Andrea (2017): Infrastructuring for Cultural Commons. In Computer Supported Cooperative Work (CSCW) 26 (1), pp. 97-133.

Plantin, Jean-Christophe; Lagoze, Carl; Edwards, Paul N.; Sandvig, Christian (2016): Infrastructure studies meet platform studies in the age of Google and Facebook. In New Media \& Society 20 (1), pp. 293-310. 
Poderi, Giacomo (2019): Sustaining platforms as commons: perspectives on participation, infrastructure, and governance. In CoDesign 15 (3), pp. 243-255.

Ross, Scott (2019): Being Real on Fake Instagram: Likes, Images, and Media Ideologies of Value. In J Linguist Anthropol 29 (3), pp. 359-374.

Srnicek, Nick (2017): Platform capitalism. Cambridge, UK, Malden, MA: Polity (Theory redux).

Taffel, Sy (2019): Digital media ecologies. Entanglements of content, code and hardware / Sy Taffel. New York, NY, USA: Bloomsbury Publishing.

van Dijck, José (2020a): Governing digital societies: Private platforms, public values. In Computer Law \& Security Review 36, p. 105377.

van Dijck, José (2020b): Seeing the forest for the trees: Visualizing platformization and its governance. In New Media \& Society.

Zuboff, Shoshana (2019): The age of surveillance capitalism. The fight for a human future at the new frontier of power. First edition. New York: PublicAffairs. 